\title{
RIGIDITY OF GRAPH PRODUCTS OF ABELIAN GROUPS
}

\author{
MAURICIO GUTIERREZ and ADAM PIGGOTT
}

(Received 19 April 2007)

\begin{abstract}
We show that if $G$ is a group and $G$ has a graph-product decomposition with finitely generated abelian vertex groups, then $G$ has two canonical decompositions as a graph product of groups: a unique decomposition in which each vertex group is a directly indecomposable cyclic group, and a unique decomposition in which each vertex group is a finitely generated abelian group and the graph satisfies the $T_{0}$ property. Our results build on results by Droms, Laurence and Radcliffe.
\end{abstract}

2000 Mathematics subject classification: primary 20E34, 20E06.

Keywords and phrases: graph products of groups.

\section{Introduction}

A labeled-graph is a pair $\left(\Gamma, \mathcal{G}_{\Gamma}\right)$, where $\Gamma$ is a non-trivial finite simplicial graph with vertex set $V_{\Gamma}$ and $\mathcal{G}_{\Gamma}=\left\{G_{u}\right\}_{u \in V_{\Gamma}}$ is a family of non-trivial groups (the vertex groups). The graph-product construction, first defined in [2], associates a group to each labeled-graph: the graph product $W\left(\Gamma, \mathcal{G}_{\Gamma}\right)$ is the quotient of the free product $*_{u \in V_{\Gamma}} G_{u}$ by relations that allow elements of $G_{u}$ and $G_{u^{\prime}}$ to commute if $u$ and $u^{\prime}$ are adjacent in $\Gamma$. The construction interpolates between the free product construction, in the case when $\Gamma$ is a discrete graph, and the direct product construction, in the case when $\Gamma$ is a complete graph. We say that the labeled-graph $\left(\Gamma, \mathcal{G}_{\Gamma}\right)$ describes $a$ graph-product decomposition of a group $G$ if $G \cong W\left(\Gamma, \mathcal{G}_{\Gamma}\right)$.

In the present article we study groups that have a graph-product decomposition with finitely generated abelian vertex groups (or, equivalently, cyclic vertex groups). A number of important classes of groups have this property, including finitely generated abelian groups, finitely generated non-abelian free groups, right-angled Coxeter groups and right-angled Artin groups (also known as 'graph groups').

A labeled-graph isomorphism $f:\left(\Gamma, \mathcal{G}_{\Gamma}\right) \rightarrow\left(\Sigma, \mathcal{G}_{\Sigma}\right)$ is a bijection $f: V_{\Gamma} \rightarrow V_{\Sigma}$ for which the following conditions hold:

(C) 2008 Australian Mathematical Society 0004-9727/08 \$A2.00 + 0.00 

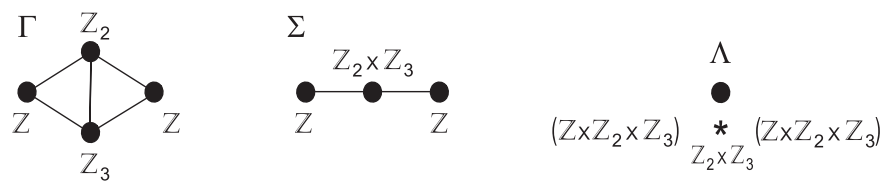

FIGURE $1 . W\left(\Gamma, \mathcal{G}_{\Gamma}\right) \cong W\left(\Sigma, \mathcal{G}_{\Sigma}\right) \cong W\left(\Lambda, \mathcal{G}_{\Lambda}\right)$.

(1) for all $u, v \in V_{\Gamma}(u, v$ adjacent in $\Gamma) \Leftrightarrow(f(u), f(v)$ adjacent in $\Sigma)$;

(2) for all $u \in V_{\Gamma} G_{u} \cong G_{f(u)}$.

We write $\left(\Gamma, \mathcal{G}_{\Gamma}\right) \cong\left(\Sigma, \mathcal{G}_{\Sigma}\right)$ in the case when a labeled-graph isomorphism exists.

The example in Figure 1 shows that non-isomorphic labeled-graphs may determine isomorphic graph products. If one wishes to study a class of groups $\mathfrak{G}$, and one hopes to take advantage of graph-product decompositions when doing so, it is desirable to identify a canonical isomorphism class of labeled-graphs $\left(\Gamma, \mathcal{G}_{\Gamma}\right)$ for each isomorphism class of groups $G \in \mathfrak{G}$. Further, the choice of labeled-graph should be sufficiently 'natural' that one may look to the structure of $\left(\Gamma, \mathcal{G}_{\Gamma}\right)$ to explain the structure of $G$. One way in which a graph-product decomposition may be considered natural is if it is minimal, in some sense, and if any two such minimal decompositions are isomorphic.

Droms [1] proved that if $G$ has a graph-product decomposition with infinite cyclic vertex groups, then any two such decompositions are isomorphic. Using different methods, Laurence [5, Isomorphism Theorem for Graph Groups, p. 329] proved a stronger statement that includes information about a particular labeled-graph isomorphism. Radcliffe [6] proved that if $G$ has a graph-product decomposition with directly indecomposable finite vertex groups, then any two such decompositions are isomorphic. Under the additional hypothesis that the vertex groups are cyclic, a close reading of Radcliffe's argument yields a result analogous to that of Laurence (Theorem 4.4). None of the work mentioned above allowed both finite and infinite vertex groups.

The main result of the present article is that if $G$ has a graph-product decomposition with directly indecomposable cyclic vertex groups, then any two such decompositions are isomorphic (Theorem 5.3). It follows that if $G$ has a graph-product decomposition with finitely generated abelian vertex groups and a graph which satisfies the $T_{0}$ property (Definition 3.1), then any two such decompositions are isomorphic (Corollary 5.4). Thus if $G$ is a group and $G$ has a graph-product decomposition with finitely generated abelian vertex groups, then $G$ has two canonical decompositions as a graph product of groups: a unique decomposition in which each vertex group is a directly indecomposable cyclic group ( $\operatorname{such}$ as $\left(\Gamma, \mathcal{G}_{\Gamma}\right)$ in Figure 1 ), and a unique decomposition in which each vertex group is a finitely generated abelian group and the graph satisfies the $T_{0}$ property (such as $\left(\Sigma, \mathcal{G}_{\Sigma}\right)$ in Figure 1$)$. The first decomposition is minimal in the sense that the vertex groups are minimal; the second decomposition 
is minimal in the sense that the graph has the least number of vertices when we allow only finitely generated abelian groups as vertex groups.

Our techniques are combinatorial. Our arguments make essential use of the results of Droms, Laurence and Radcliffe mentioned above. In Section 2 we discuss some preliminary results on graphs and graph products, in Section 3 we remind the reader of the $T_{0}$ property and the $T_{0}$-quotient of a labeled-graph as used by Radcliffe, in Section 4 we state a result by Laurence and sharpen a result by Radcliffe and in Section 5 we prove the main result.

\section{Graphs and graph products}

For a non-trivial finite simplicial graph $\Lambda$ (that is, a graph with no circuits of length less than three), we write $V_{\Lambda}$ for the set of vertices of $\Lambda$. The subgraph of $\Lambda$ determined by a subset $U \subseteq V_{\Lambda}$ is the full subgraph of $\Gamma$ determined by the vertices in $U$. We write $\operatorname{MCS}(\Lambda)$ for the set of maximal complete subgraphs (or cliques) of $\Lambda$.

Let $\left(\Lambda, \mathcal{G}_{\Lambda}\right)$ be a labeled-graph. Each full subgraph $\Theta$ of $\Lambda$ determines a labeledsubgraph $\left(\Theta, \mathcal{G}_{\Theta}\right)$. We write $W(\Theta)$ for the subgroup of $W\left(\Lambda, \mathcal{G}_{\Lambda}\right)$ generated by the natural image of the set $\bigcup_{u \in V_{\Theta}} G_{u}$, and we note that $W(\Theta) \cong W\left(\Theta, \mathcal{G}_{\Theta}\right)$. In particular, we shall often write $W(\Lambda)$ for $W\left(\Lambda, \mathcal{G}_{\Lambda}\right)$.

A cyclic group is primary if it has prime-power order, and directly indecomposable if it has infinite order or it is primary. For the remainder of this section we assume that $\mathcal{G}_{\Lambda}$ is a family of directly indecomposable cyclic groups. Following common practice, we abuse notation by ignoring the formal distinction between the elements of $V_{\Lambda}$ and the generators of $W(\Lambda)$.

We write $\Lambda_{T}$ for the subgraph of $\Lambda$ determined by the vertices $u \in V_{\Lambda}$ for which $G_{u}$ has finite order and we write $\Lambda_{A}$ for the subgraph of $\Lambda$ determined by the vertices $u \in V_{\Lambda}$ for which $G_{u}$ has infinite order (we have selected $T$ for torsion and $A$ for Artin). The next lemma follows immediately from a more general result by Green [2, Theorem 3.26].

LEMMA 2.1. If $\mathcal{G}_{\Lambda}$ is a family of directly indecomposable cyclic groups, then each maximal finite subgroup of $W(\Lambda)$ is abelian and

$$
\left\{W(\Theta) \mid \Theta \in \operatorname{MCS}\left(\Lambda_{T}\right)\right\}
$$

is a complete set of representatives for the conjugacy classes of maximal finite subgroups of $W(\Lambda)$.

Following [4, 5], a word $w$ in the alphabet $V_{\Lambda}^{ \pm 1}$ is said to be reduced if there is no shorter word $w^{\prime}$ which defines the same element of $W(\Lambda)$. For a group element $g \in W(\Lambda)$ and a word $w$ in the alphabet $V_{\Lambda}^{ \pm 1}$, we write $g \stackrel{\text { red }}{=} w$ if $w$ is a reduced word which equals $g$ in the group $W(\Lambda)$. For words $w$ and $w^{\prime}$ in the alphabet $V_{\Lambda}^{ \pm 1}$, we write $w \equiv w^{\prime}$ if $w$ and $w^{\prime}$ are equal as words and we say that $w$ is transformed into $w^{\prime}$ by a 
letter swapping operation if $w \equiv w_{1} u^{\epsilon} v^{\delta} w_{2}$ and $w^{\prime} \equiv w_{1} v^{\delta} u^{\epsilon} w_{2}$ for some adjacent vertices $u, v \in V_{\Lambda}$, some exponents $\epsilon, \delta \in\{ \pm 1\}$ and some reduced words $w_{1}, w_{2}$. Laurence showed that for vertices $v_{1}, \ldots, v_{p} \in V_{\Lambda}$ and integers $\epsilon_{1}, \ldots, \epsilon_{p} \in\{ \pm 1\}$, if $w \equiv v_{1}^{\epsilon_{1}} \ldots v_{p}^{\epsilon_{p}}$ is not reduced, then there exists $1 \leq i<j \leq p$ such that $v_{i}=v_{j}$, $\epsilon_{i}+\epsilon_{j}=0$ and $v_{i}$ is adjacent to each of the vertices $v_{i+1}, \ldots, v_{j-1}$ (the Deletion Condition) [4, Corollary 3.1.1]. It follows from the Normal Form Theorem for Graph Products [2] (see also [4]) that if two reduced words $w, w^{\prime}$ define the same element of $W(\Lambda)$, then $w$ can be transformed into $w^{\prime}$ by a finite number of letter swapping operations (the Transpose Condition). It follows from the Transpose Condition that we may define

$$
\begin{gathered}
\operatorname{supp}(g):=\left\{u \in V_{\Lambda} \mid u \text { or } u^{-1} \text { appears in some reduced word for } g\right\}, \\
\operatorname{csupp}(g):=\left\{u \in V_{\Lambda} \mid \forall w \in W(\Lambda) u \in \operatorname{supp}\left(w g w^{-1}\right)\right\} .
\end{gathered}
$$

We say that $d$ is a terminal segment of $w$ if $d$ is a reduced word and there exists a reduced word $w^{\prime}$ such that $w \equiv w^{\prime} d$.

For an element $g \in W(\Lambda)$, we write $C_{W(\Lambda)}(g)$ for the centralizer of $g$ in $W(\Lambda)$. An element $u \in W(\Lambda)$ is said to be a CP element (for commuting product) if there exists a complete subgraph $\Delta \subseteq \Gamma$ such that $u \in W(\Delta)$. We write $u^{\star}$ for the subgraph of $\Gamma$ generated by $\operatorname{supp}(u)$ and those vertices adjacent to each vertex in $\operatorname{supp}(u)$. The centralizer of a CP element in $W(\Lambda)$ has a particularly simple form.

LEMMA 2.2. If $\mathcal{G}_{\Lambda}$ is a family of directly indecomposable cyclic groups and $u$ is a $C P$ element of $W(\Lambda)$, then $C_{W(\Lambda)}(u)=W\left(u^{\star}\right)$.

PROOF. Let $u$ be a CP element of $W(\Lambda)$. We shall abuse notation by also writing $u$ for a reduced word representing the group element $u$. It is clear that $W\left(u^{\star}\right) \subset C_{W(\Lambda)}(u)$.

Suppose that $C_{W(\Lambda)}(u)-W\left(u^{\star}\right)$ is non-empty and let $g$ be a minimal length (with respect to the generating set $\left.V_{\Gamma}^{ \pm 1}\right)$ element of $C_{W(\Lambda)}(u)-W\left(u^{\star}\right)$. The minimality of $g$ implies that no terminal segment of $g$ is contained in $W\left(u^{\star}\right)$, so $g \stackrel{\text { red }}{=} w^{\prime} y^{\epsilon}$ for some vertex $y \notin u^{\star}$, some exponent $\epsilon \in\{ \pm 1\}$ and some reduced word $w^{\prime}$.

We claim that $w^{\prime} y^{\epsilon} u$ is a reduced word. Suppose that $w^{\prime} y^{\epsilon} u$ is not a reduced word. It follows from the Deletion Condition that some sequence of letter swapping operations will transform the word $w^{\prime} y^{\epsilon}$ into a word $w^{\prime \prime} v^{\delta}$, where $v \in \operatorname{supp}(u)$, $\delta \in\{ \pm 1\}$ and $v^{-\delta}$ appears in $u$. But then $g$ has a terminal segment in $W\left(u^{\star}\right)$. This contradiction completes the proof of the claim.

By hypothesis, $w^{\prime} y^{\epsilon} u=u w^{\prime} y^{\epsilon}$ and it follows that $u w^{\prime} y^{\epsilon}$ is also a reduced word. By the transpose condition, $w^{\prime} y^{\epsilon} u$ may be transformed to $u w^{\prime} y^{\epsilon}$ by a finite number of letter swapping operations. Since $y^{\epsilon} \notin u^{\star}$, we have that $y \notin \operatorname{supp}(u)$. It follows that, to transform $w^{\prime} y^{\epsilon} u$ to $u w^{\prime} y^{\epsilon}$ by letter swapping operations, we must have that $y$ is adjacent to each vertex in $\operatorname{supp}(u)$. But this contradicts the hypothesis that $y \notin u^{\star}$. 


\section{An equivalence relation on the vertices of a graph}

In this section we remind the reader of an equivalence relation on the vertices of a graph which proved to be a key idea in [6].

Let $\left(\Lambda, \mathcal{G}_{\Lambda}\right)$ be a labeled-graph. Following [6], we define a relation $\sim_{\Lambda}$ on the set $V_{\Lambda}$ as follows:

$$
u \sim_{\Lambda} v \Leftrightarrow(\forall \Theta \in \operatorname{MCS}(\Lambda) u \in \Theta \Leftrightarrow v \in \Theta) .
$$

It is easily verified that $\sim_{\Lambda}$ is an equivalence relation. We write $\tilde{u}$ for the $\sim_{\Lambda}$ equivalence class of $u \in V_{\Lambda}$. It is immediate from the definitions that each $\sim_{\Lambda}$ equivalence class determines a complete subgraph of $\Lambda$.

DEFinition 3.1. If each $\sim_{\Lambda}$ equivalence class is a singleton set, then we say that $\Lambda$ satisfies the $T_{0}$ property.

The relation $\sim_{\Lambda}$ determines a well-defined quotient of $\Lambda$ and a well-defined quotient of $\left(\Lambda, \mathcal{G}_{\Lambda}\right)$.

Definition 3.2. Let $\Lambda_{0}$ denote the graph with vertex set $V_{\Lambda_{0}}$ in one-to-one correspondence with the $\sim_{\Lambda}$ equivalence classes of $V_{\Lambda}$ and with adjacency determined by the following rule:

$\tilde{u}$ and $\tilde{v}$ adjacent in $\Lambda_{0} \Leftrightarrow u$ and $v$ adjacent in $\Lambda$ and $u \psi_{\Lambda} v$.

For each $\tilde{u} \in V_{\Lambda_{0}}$, define $G_{\tilde{u}}:=\times_{v \in \tilde{u}} G_{v}$. Write $\mathcal{G}_{\Lambda_{0}}:=\left\{G_{\tilde{u}}\right\}_{\tilde{u} \in V_{\Lambda_{0}}}$. The graph $\Lambda_{0}$ is called the $T_{0}$-quotient of $\Lambda$ and the labeled-graph $\left(\Lambda_{0}, \mathcal{G}_{\Lambda_{0}}\right)$ is called the $T_{0}$-quotient of $\left(\Lambda, \mathcal{G}_{\Lambda}\right)$.

We record some observations on the $T_{0}$-quotients $\Lambda_{0}$ and $\left(\Lambda_{0}, \mathcal{G}_{\Lambda_{0}}\right)$.

LEMMA 3.3. Let $\left(\Lambda, \mathcal{G}_{\Lambda}\right)$ be a labeled-graph and let $\left(\Lambda_{0}, \mathcal{G}_{\Lambda_{0}}\right)$ be its $T_{0}$-quotient. The following properties hold:

(1) $\Lambda_{0}$ satisfies the $T_{0}$ property;

(2) vertices $u, v \in V_{\Lambda}$ are adjacent in $\Lambda$ if and only if either $\tilde{u}$ and $\tilde{v}$ are adjacent in $\Lambda_{0}$ or $\tilde{u}=\tilde{v}$;

(3) $W\left(\Lambda, \mathcal{G}_{\Lambda}\right) \cong W\left(\Lambda_{0}, \mathcal{G}_{\Lambda_{0}}\right)$.

We now establish that in the case that $\mathcal{G}_{\Lambda}$ is a family of directly indecomposable cyclic groups, the isomorphism class of $\left(\Lambda, \mathcal{G}_{\Lambda}\right)$ is uniquely determined by the isomorphism class of the $T_{0}$-quotient $\left(\Lambda_{0}, \mathcal{G}_{\Lambda_{0}}\right)$.

LEMMA 3.4. Let $\left(\Lambda, \mathcal{G}_{\Lambda}\right)$ and $\left(\Xi, \mathcal{G}_{\Xi}\right)$ be labeled-graphs with directly indecomposable cyclic vertex groups. Then $\left(\Lambda, \mathcal{G}_{\Lambda}\right)$ and $\left(\Xi, \mathcal{G}_{\Xi}\right)$ are isomorphic if and only if $\left(\Lambda_{0}, \mathcal{G}_{\Lambda_{0}}\right)$ and $\left(\Xi_{0}, \mathcal{G}_{\Xi_{0}}\right)$ are isomorphic.

PROOF. It is clear that each isomorphism $\left(\Lambda, \mathcal{G}_{\Lambda}\right) \rightarrow\left(\Xi, \mathcal{G}_{\Xi}\right)$ induces an isomorphism $\left(\Lambda_{0}, \mathcal{G}_{\Lambda_{0}}\right) \rightarrow\left(\Xi_{0}, \mathcal{G}_{\Xi_{0}}\right)$. Now suppose that there exists an isomorphism $t_{0}:\left(\Lambda_{0}, \mathcal{G}_{\Lambda_{0}}\right) \rightarrow\left(\Xi_{0}, \mathcal{G}_{\Xi_{0}}\right)$. Each element of $\mathcal{G}_{\Lambda_{0}}$ (respectively $\mathcal{G}_{\Xi_{0}}$ ) is a finitely 
generated abelian group. It is well known that such groups have a unique decomposition as a direct product of directly indecomposable cyclic groups. Thus, for each $\tilde{u} \in V_{\Lambda_{0}}$, there exists a labeled-graph isomorphism $t_{\tilde{u}}$ from the subgraph of $\Lambda$ generated by the vertices in $\tilde{u}$ to the subgraph of $\Xi$ generated by the vertices in $t_{0}(\tilde{u})$. Define $t$ to be the bijection $V_{\Lambda} \rightarrow V_{\Xi}$ which restricts to $t_{\tilde{u}}$ for each equivalence class $\tilde{u} \in V_{\Lambda_{0}}$. It follows from the definitions and Lemma 3.3(2) that $t$ is a labeled-graph isomorphism.

\section{Some preliminary rigidity results}

For the remainder of this paper we assume that $\left(\Gamma, \mathcal{G}_{\Gamma}\right)$ and $\left(\Sigma, \mathcal{G}_{\Sigma}\right)$ are labeledgraphs with directly indecomposable cyclic vertex groups. As in Section 2, we shall abuse notation by ignoring the formal distinction between the elements of $V_{\Gamma}$ (respectively $V_{\Sigma}$ ) and the generators of $W(\Gamma)$ (respectively $W(\Sigma)$ ).

As stated in the introduction, Droms [1] proved that if $G$ has a graph-product decomposition with infinite cyclic vertex groups, then any two such decompositions are isomorphic. Laurence proved the following stronger statement using peak reduction techniques.

THEOREM 4.1 (Laurence [5, p. 329]). If $\mathcal{G}_{\Gamma}$ and $\mathcal{G}_{\Sigma}$ are families of infinite cyclic groups and $\alpha: W(\Gamma) \rightarrow W(\Sigma)$ is an isomorphism, then there exists a labeled-graph isomorphism $a:\left(\Gamma, \mathcal{G}_{\Gamma}\right) \rightarrow\left(\Sigma, \mathcal{G}_{\Sigma}\right)$ for which the following property holds:

$$
\text { for all } u \in V_{\Gamma}, a(u) \in \operatorname{csupp}(\alpha(u)) \text {. }
$$

Also, as stated in the introduction, Radcliffe [6] proved that if $G$ has a graph-product decomposition with directly indecomposable finite vertex groups, then any two such decompositions are isomorphic. In this section we work towards a full analogue of Theorem 4.1, under the hypothesis of primary cyclic vertex groups.

By Lemma 2.1, if $\mathcal{G}_{\Lambda}$ is a family of directly indecomposable cyclic groups and $g \in W(\Lambda)$ is an element of finite order, then there exists a unique element $[g]$ of minimal length in the conjugacy class of $g$ and there exists $\Theta \in \operatorname{MCS}\left(\Lambda_{T}\right)$ such that $\operatorname{csupp}(g) \subseteq \Theta$ and $[g] \in W(\Theta)$. This fact plays a central role in the present article because of the following lemma.

LEMMA 4.2. If $\mathcal{G}_{\Gamma}$ and $\mathcal{G}_{\Sigma}$ are families of primary cyclic groups and $\tau: W(\Gamma) \rightarrow$ $W(\Sigma)$ is an isomorphism, then the map $\hat{\tau}: V_{\Gamma} \rightarrow W(\Sigma)$ determined by the rule $u \mapsto[\tau(u)]$ extends to an isomorphism $\hat{\tau}: W(\Gamma) \rightarrow W(\Sigma)$.

PROOF. Consider the presentation of $W(\Gamma)$ implicit in the graph-product decomposition $\left(\Gamma, \mathcal{G}_{\Gamma}\right)$. We shall show that $\hat{\tau}$ extends to a homomorphism $W(\Gamma) \rightarrow$ $W(\Sigma)$ by checking that the relations in this presentation of $W(\Gamma)$ are 'preserved' by $\hat{\tau}$. Since $\tau$ is an isomorphism and conjugation preserves the order of an element, it 
is clear that the order of each vertex is preserved by $\hat{\tau}$. If $u, v \in V_{\Gamma}$ are adjacent in $\Gamma$, then $\langle u, v\rangle$ has finite order and so does $\langle\tau(u), \tau(v)\rangle$. By Lemma 2.1, there exists $\Theta \in \operatorname{MCS}(\Sigma)$ and $w \in W(\Sigma)$ such that $\tau(u), \tau(v) \in w W(\Theta) w^{-1}$. Then $\hat{\tau}(u), \hat{\tau}(v)$ are contained in the abelian subgroup $W(\Theta)$. Thus $\hat{\tau}(u) \hat{\tau}(v) \hat{\tau}(u)^{-1} \hat{\tau}(v)^{-1}=1$ and the relation $u v u^{-1} v^{-1}$ is preserved by $\hat{\tau}$.

We shall show that the homomorphism $\hat{\tau}$ is an isomorphism by showing that $\widehat{\tau^{-1}} \circ$ $\hat{\tau}(v)=v$ for each $v \in V_{\Gamma}$. Let $v \in V_{\Gamma}$. There exists $\Theta \in \operatorname{MCS}(\Sigma), a \in W(\Theta)$ and $w \in W(\Sigma)$ such that $\tau(v)=w a w^{-1}$ and $\hat{\tau}(v)=a$. Then $\tau^{-1}(a)=\tau^{-1}\left(w^{-1}\right) v \tau^{-1}(w)$ and $\widehat{\tau^{-1}}(a)=v$. So $\widehat{\tau^{-1}} \circ \hat{\tau}(v)=\widehat{\tau^{-1}}(a)=v$, as required.

We also require the following result concerning the isomorphisms of finite abelian groups.

LEMMA 4.3. If $\Gamma$ and $\Sigma$ are complete graphs and $\mathcal{G}_{\Gamma}$ and $\mathcal{G}_{\Sigma}$ are families of primary cyclic groups and $\tau: W(\Gamma) \rightarrow W(\Sigma)$ is an isomorphism, then there exists a labeled-graph isomorphism $t:\left(\Gamma, \mathcal{G}_{\Gamma}\right) \rightarrow\left(\Sigma, \mathcal{G}_{\Sigma}\right)$ for which the following property holds:

$$
\text { for all } u \in V_{\Gamma}, t(u) \in \operatorname{supp}(\tau(u))
$$

PROOF. Without loss of generality we may assume that $\mathcal{G}_{\Gamma}$ and $\mathcal{G}_{\Sigma}$ are families of $p$-primary cyclic groups for a fixed prime $p$.

Let $V_{\Gamma}=\left\{g_{1}, g_{2}, \ldots, g_{k}\right\}$, let $V_{\Sigma}=\left\{s_{1}, s_{2}, \ldots, s_{k}\right\}$ and let $A=\left(a_{\ell m}\right)$ be the matrix of integers such that $s_{m}$ appears with exponent sum $a_{\ell m}$ in $\tau\left(g_{\ell}\right)$. Recall that

$$
\operatorname{det} A=\sum_{\sigma \in \operatorname{Sym}(n)} \operatorname{sgn}(\sigma) a_{1 \sigma(1)} a_{2 \sigma(2)} \ldots a_{k \sigma(k)},
$$

where $\operatorname{Sym}(n)$ denotes the symmetric group on the set $\{1,2, \ldots, n\}$. By [3, Theorem 3.6], $A(\bmod p) \in \mathrm{GL}_{k}\left(\mathbb{F}_{p}\right)$. Hence $\operatorname{det} A$ is not divisible by $p$ and at least one term of the sum (4.1) is not divisible by $p$. Thus there exists $\sigma \in \operatorname{Sym}(n)$ such that $a_{m \sigma(m)}$ is non-trivial modulo $p$ for each $m$; hence $s_{\sigma(m)} \in \operatorname{supp}\left(\tau\left(g_{m}\right)\right)$ for each $m$. Define $t\left(g_{m}\right)=s_{\sigma(m)}$ for each $1 \leq m \leq k$. By construction, $t$ has the required properties.

We now give an analogue of Theorem 4.1. The proof below is an interpretation of Radcliffe's argument [6], with Lemma 4.3 applied at the appropriate place to strengthen the result.

THEOREM 4.4 (Radcliffe [6]). If $\mathcal{G}_{\Gamma}$ and $\mathcal{G}_{\Sigma}$ are families of primary cyclic groups and $\tau: W(\Gamma) \rightarrow W(\Sigma)$ is an isomorphism, then there exists a labeled-graph isomorphism $t:\left(\Gamma, \mathcal{G}_{\Gamma}\right) \rightarrow\left(\Sigma, \mathcal{G}_{\Sigma}\right)$ for which the following property holds:

$$
\text { for all } u \in V_{\Gamma} t(u) \in \operatorname{csupp}(\tau(u)) \text {. }
$$


PRoOF. In this paragraph we define a map $t_{0}: V_{\Gamma_{0}} \rightarrow V_{\Sigma_{0}}$. Let $\tilde{u} \in V_{\Gamma_{0}}$. It follows from the $T_{0}$ property that $\tilde{u} \in V_{\Gamma_{0}}$ is uniquely identified by its memberships and non-memberships in elements of $\operatorname{MCS}\left(\Gamma_{0}\right)$. That is, the singleton set $\{\tilde{u}\}$ is the intersection of the maximal complete subgraphs of $\Gamma_{0}$ which contain $\tilde{u}$ minus the union of the maximal complete subgraphs of $\Gamma_{0}$ which do not contain $\tilde{u}$. It follows from the definition that $\hat{\tau}$ determines a one-to-one correspondence between the sets $\operatorname{MCS}\left(\Gamma_{0}\right)$ and $\operatorname{MCS}\left(\Sigma_{0}\right)$. Thus $\hat{\tau}\left(G_{\tilde{u}}\right)$ may be written as an intersection of elements in $\operatorname{MCS}\left(\Sigma_{0}\right)$ minus a union of elements in $\operatorname{MCS}\left(\Sigma_{0}\right)$. The $T_{0}$ property then implies that $\hat{\tau}\left(G_{\tilde{u}}\right)=G_{\tilde{v}}$ for some $\tilde{v} \in V_{\Sigma_{0}}$. Define $t_{0}(\tilde{u})=\tilde{v}$.

From the definitions (or using Lemma 2.2), the reader may confirm that $t_{0}$ is a labeled-graph isomorphism and that $\hat{\tau}$ restricts to an isomorphism $W\left(G_{\tilde{u}}\right) \rightarrow$ $W\left(G_{t_{0}(\tilde{u})}\right)$ for each $\tilde{u} \in V_{\Gamma_{0}}$. Following the proof of Lemma 3.4, we may lift $t_{0}$ to a labeled-graph isomorphism $t:\left(\Gamma, \mathcal{G}_{\Gamma}\right) \rightarrow\left(\Sigma, \mathcal{G}_{\Sigma}\right)$.

Now, recall that each $\sim_{\Gamma}$ (respectively $\sim_{\Sigma}$ ) equivalence class of vertices $\tilde{u}$ determines a complete subgraph of $\Gamma$ (respectively $\Sigma$ ) and hence a finite abelian subgroup of $W(\Gamma)$ (respectively $(W(\Sigma))$. By Lemma 4.3, we may choose the lift $t$ of $t_{0}$ so that, on each subgraph of $\Gamma$ determined by a single equivalence class $\tilde{u}$ of vertices, $t$ restricts to a labeled-graph isomorphism with the property that $t(u) \in \operatorname{supp}(\hat{\tau}(u))$ for each $u \in \tilde{u}$. It follows that $t(u) \in \operatorname{csupp}(\tau(u))$ for each $u \in V_{\Gamma}$.

\section{The main theorem}

We remind the reader that $\left(\Gamma, \mathcal{G}_{\Gamma}\right)$ and $\left(\Sigma, \mathcal{G}_{\Sigma}\right)$ are labeled-graphs with directly indecomposable cyclic vertex groups. We now assume that there exists a group isomorphism $\phi: W(\Gamma) \rightarrow W(\Sigma)$. Our task is to exhibit a labeled-graph isomorphism $\left(\Gamma, \mathcal{G}_{\Gamma}\right) \rightarrow\left(\Sigma, \mathcal{G}_{\Sigma}\right)$

Let $T(\Gamma)$ (respectively $T(\Sigma)$ ) denote the subgroup of $W(\Gamma)$ (respectively $W(\Sigma)$ ) generated by the elements of finite order. Let $\rho_{\Sigma_{A}}: W(\Sigma) \rightarrow W\left(\Sigma_{A}\right)$ denote the retraction homomorphism determined by

$$
\text { for all } u \in V_{\Sigma}, u \mapsto \begin{cases}u & \text { if } u \in V_{\Sigma_{A}}, \\ 1 & \text { if } u \in V_{\Sigma_{T}}\end{cases}
$$

LeMma 5.1. Let $\alpha: V_{\Gamma_{A}} \rightarrow W\left(\Sigma_{A}\right)$ be defined by $v \mapsto \rho_{\Sigma_{A}} \circ \phi(v)$. Then $\alpha$ extends to an isomorphism $\alpha: W\left(\Gamma_{A}\right) \rightarrow W\left(\Sigma_{A}\right)$.

ProOf. Since $\phi(T(\Gamma))=T(\Sigma)$, the isomorphism $\phi: W(\Gamma) \rightarrow W(\Sigma)$ induces an isomorphism $\hat{\phi}: W(\Gamma) / T(\Gamma) \rightarrow W(\Sigma) / T(\Sigma)$. Since $W\left(\Gamma_{A}\right) \cap T(\Gamma)=\{1\}$ and $V_{\Gamma_{T}} \subset T(\Gamma)$, the quotient map $W(\Gamma) \rightarrow W(\Gamma) / T(\Gamma)$ restricts to an isomorphism $\pi_{\Gamma_{A}}: W\left(\Gamma_{A}\right) \rightarrow W(\Gamma) / T(\Gamma)$. Similarly, the quotient map $W(\Sigma) \rightarrow W(\Sigma) / T(\Sigma)$ restricts to an isomorphism $\pi_{\Sigma_{A}}: W\left(\Sigma_{A}\right) \rightarrow W(\Sigma) / T(\Sigma)$. Thus $\left(\pi_{\Sigma_{A}}\right)^{-1} \circ \hat{\phi} \circ \pi_{\Gamma_{A}}$ is an isomorphism $W\left(\Gamma_{A}\right) \rightarrow W\left(\Sigma_{A}\right)$. Calculation confirms that $\left(\pi_{\Sigma_{A}}\right)^{-1} \circ \hat{\phi} \circ \pi_{\Gamma_{A}}$ $=\rho_{\Sigma_{A}} \circ \phi$. 
Recall that, for an element $w$ of finite order in $W(\Sigma)$, we write $[w]$ for the unique element of minimal length in the conjugacy class of $w$. As with Lemma 4.2, the following lemma may be verified by elementary means.

LEMMA 5.2 (Compare with Lemma 4.2). Let $\tau: V_{\Gamma_{T}} \rightarrow W\left(\Sigma_{T}\right)$ be defined by $u \mapsto[\phi(u)]$. Then $\tau$ extends to an isomorphism $\tau: W\left(\Gamma_{T}\right) \rightarrow W\left(\Sigma_{T}\right)$.

Lemmas 5.1 and 5.2 allow us to use Theorems 4.1 and 4.4 to prove our main result.

THEOREM 5.3. If $\left(\Gamma, \mathcal{G}_{\Gamma}\right)$ and $\left(\Sigma, \mathcal{G}_{\Sigma}\right)$ are labeled-graphs with directly indecomposable cyclic vertex groups and there exists a group isomorphism $\phi: W(\Gamma) \rightarrow W(\Sigma)$, then there exists a labeled-graph isomorphism $f:\left(\Gamma, \mathcal{G}_{\Gamma}\right) \rightarrow$ $\left(\Sigma, \mathcal{G}_{\Sigma}\right)$.

PROOF. By Lemma 5.1 and Theorem 4.1, there exists a labeled-graph isomorphism $a:\left(\Gamma_{A}, \mathcal{G}_{\Gamma_{A}}\right) \rightarrow\left(\Sigma_{A}, \mathcal{G}_{\Sigma_{A}}\right)$ as in the statement of Theorem 4.1. By Lemma 5.2 and Theorem 4.4, there exists a labeled-graph isomorphism $t:\left(\Gamma_{T}, \mathcal{G}_{\Gamma_{T}}\right) \rightarrow\left(\Sigma_{T}, \mathcal{G}_{\Sigma_{T}}\right)$ as in the statement of Theorem 4.4. Define $f: V_{\Gamma} \rightarrow V_{\Sigma}$ to be the bijection

$$
u \mapsto \begin{cases}a(u) & \text { if } u \in V_{\Gamma_{A}}, \\ t(u) & \text { if } u \in V_{\Gamma_{T}} .\end{cases}
$$

We claim that $f$ is a labeled-graph isomorphism.

It is immediate from the definitions that $G_{u} \cong G_{f(u)}$ for each $u \in V_{\Gamma}$. It remains to show only that $f$ preserves the structure of $\Gamma$. Since we know $a$ and $t$ to be labeledgraph isomorphisms, it remains to show only that $f$ preserves adjacency between vertices in $V_{\Gamma_{A}}$ and vertices in $V_{\Gamma_{T}}$.

Let $u \in V_{\Gamma_{T}}$ and $v \in V_{\Gamma_{A}}$ be adjacent in $\Gamma$. Since $u$ has finite order in $W(\Gamma)$, $\phi(u)$ has finite order in $W(\Sigma)$. By Lemma 2.1, there exists an inner automorphism $\iota$ of $W(\Sigma)$ such that $\iota \circ \phi(u)$ is a CP element of $W(\Sigma)$. Write $x:=\iota \circ \phi(u)$ and $y:=\iota \circ \phi(v)$. Since $x$ and $y$ commute and $x$ is a CP element of $W(\Sigma)$, we have by Lemma 2.2 that $y \in W\left(x^{\star}\right)$. Hence $\operatorname{supp}(y) \subset x^{\star}$. But $f(u)=t(u) \in \operatorname{csupp}(\phi(u))=$ $\operatorname{supp}(x)$ and $f(v)=a(v) \in \operatorname{csupp}(\phi(v))=\operatorname{csupp}(y) \subset \operatorname{supp}(y)$. Hence $f(u)$ and $f(v)$ are adjacent in $\Sigma$.

It follows from the above paragraph that $\Sigma$ has at least as many edges as $\Gamma$. Similarly, by considering $\phi^{-1}$ we may show that $\Gamma$ has at least as many edges as $\Sigma$, and hence the edges of $\Gamma$ and the edges of $\Sigma$ are in one-to-one correspondence. It follows that if $u \in V_{\Gamma_{T}}$ and $v \in V_{\Gamma_{A}}$ are not adjacent in $\Gamma$, then $t(u)$ and $a(v)$ are not adjacent in $\Sigma$.

Thus $f$ preserves the structure of $\Gamma$ and $f$ is a labeled-graph isomorphism.

Corollary 5.4. If $\left(\Gamma, \mathcal{G}_{\Gamma}\right)$ and $\left(\Sigma, \mathcal{G}_{\Sigma}\right)$ are labeled-graphs with directly indecomposable cyclic vertex groups and there exists a group isomorphism $\phi: W(\Gamma) \rightarrow W(\Sigma)$, then there exists a labeled-graph isomorphism $f_{0}:\left(\Gamma_{0}, \mathcal{G}_{\Gamma_{0}}\right) \rightarrow$ $\left(\Sigma_{0}, \mathcal{G}_{\Sigma_{0}}\right)$. 
PROOF. The result follows immediately from Theorem 5.3 and Lemma 3.4.

\section{Acknowledgements}

The authors wish to thank George McNinch for a number of helpful conversations during the preparation of this article.

\section{References}

[1] C. Droms, 'Isomorphisms of graph groups', Proc. Amer. Math. Soc. 100(3) (1987), 407-408.

[2] E. R. Green, 'Graph products of groups', PhD Thesis, The University of Leeds, 1990.

[3] C. J. Hillar and D. L. Rhea, 'Automorphisms of finite abelian groups', Amer. Math. Monthly 114(10) (2007), 917-923.

[4] M. R. Laurence, 'Automorphisms of graph products of groups', PhD Thesis, Queen Mary College, University of London, 1993.

[5] _ 'A generating set for the automorphism group of a graph group', J. London Math. Soc. (2) 52(2) (1995), 318-334.

[6] D. G. Radcliffe, 'Rigidity of graph products of groups', Algebr. Geom. Topol. 3 (2003), 1079-1088 (electronic).

MAURICIO GUTIERREZ, Department of Mathematics, Tufts University, 503 Boston Ave, Medford, MA 02155, USA

e-mail: Mauricio.Gutierrez@tufts.edu

ADAM PIGGOTT, Department of Mathematics, Tufts University, 503 Boston Ave, Medford, MA 02155, USA

e-mail: Adam.Piggott@tufts.edu 